\title{
Aplicabilidad del componente de nutrición y alimentación de la cátedra de salud pública en una institución educativa pública de Pamplona
}

\author{
Applicability of the nutrition and food component of the chair of public health in a public educational institution in \\ Pamplona
}

Irene Botía-Rodríguez' orcid.org/0000-0001-9862-3115

Lennys Carvajal-Suárez ${ }^{*}$ orcid.org/0000-0001-5591-3155

María Paola Molina-Rúas' orcid.org/0000-0001-6490-7670

1 Facultad de Salud, Universidad de Pamplona. Norte de Santander, Colombia.

Botía-Rodríguez I, Carvajal-Suárez L, Molina-Rúas MP. Aplicabilidad del componente de nutrición y alimentación de la cátedra de salud pública en una institución educativa pública de Pamplona. Univ. Salud. 2018;20(3):247-254. DOI: http://dx.doi.org/10.22267/rus.182003.127

\section{Resumen}

Introducción: La cátedra de salud pública ha sido implementada sostenible e integralmente para generar estilos de vida saludable y contribuir con el mejoramiento de la calidad de vida. Objetivo: Evaluar la aplicabilidad del componente de nutrición y alimentación de la cátedra de salud pública en una institución educativa pública de Pamplona. Materiales y métodos: Se realizó un estudio descriptivo en 108 estudiantes de grados $7^{\circ}$ a $11^{\circ}$ en quienes se aplicó una encuesta con preguntas de escala tipo Likert. Resultados: $40 \%$ de los estudiantes refirió que siempre procuran tener una alimentación balanceada y saludable a fin de evitar trastornos alimentarios; el 63\% consideró que los conocimientos adquiridos sobre lactancia materna nunca han sido aplicados en el entorno en el cual se desarrollan. Pese a ello, las mujeres aplican con mayor frecuencia los conocimientos adquiridos con respecto a los hombres $(\mathrm{p}=0,031)$. Los estudiantes de séptimo grado procuran tener una alimentación balanceada y saludable a fin de evitar trastornos alimentarios con respecto a los demás grados escolares $(\mathrm{p}=0,029)$. Conclusiones: Se identifican falencias en la aplicabilidad de los conocimientos en nutrición y alimentación, por lo cual es necesario implementar estrategias educativas efectivas para favorecer la adopción de prácticas saludables en los adolescentes.

Palabras clave: Salud pública; educación en salud; educación alimentaria y nutricional. (Fuente: DeCS, Bireme).

\begin{abstract}
Introduction: The chair of public health has been implemented in a sustainable and integral way to generate healthy lifestyles and contribute to improving the quality of life. Objective: To evaluate the applicability of the nutrition and food component of the chair of public health in a public educational institution in Pamplona. Materials and methods: A descriptive study was conducted in 108 students from 7 to 11 grades, who answered a survey with Likert-type questions. Results: $40 \%$ of the students said they always try to have a balanced and healthy diet in order to avoid eating disorders. $63 \%$ considered that the knowledge acquired on breastfeeding has never been applied in the environment in which they develop. In spite of this, women more frequently apply the knowledge acquired with respect to men $(\mathrm{P}=0.031)$. Seventh graders try to have a balanced and healthy diet to avoid eating disorders with respect to other school grades $(\mathrm{P}=0.029)$. Conclusions: Flaws are identified in the applicability of knowledge in nutrition and food, so it is necessary to implement effective educational strategies to encourage the adoption of healthy practices in adolescents.
\end{abstract}

Key words: Public health; health education; food and nutritional education. (Source: DeCS, Bireme). 


\section{Introducción}

La educación para la salud se concibe como un proceso de orden intelectual, psicológico y social, que comprende actividades destinadas a incrementar la aptitud de los individuos a tomar decisiones que afectan el bienestar personal, familiar y social(1). Se considera cualquier combinación de actividades de información y educación que conduce a las personas a desear estar sanos, a saber cómo alcanzar la salud y mantenerla(2). La educación para la salud se trata fundamentalmente de una actividad educativa diseñada para aumentar el conocimiento de la población en relación con la salud y desarrollar actitudes, hábitos y estilos de vida que la promuevan(3). En Colombia, la promoción de la salud, empezó a cobrar vitalidad a comienzos del decenio de los noventa, cuando se establecieron los principios formulados en la Carta de Ottawa de 1986, generando las bases que han servido como marco de referencia para la acción y las cinco áreas de actuación que hasta el momento han guiado la labor de la promoción de salud(4).

La educación en nutrición, entendida como la combinación de experiencias de aprendizaje, diseñadas para facilitar la adopción voluntaria de conductas alimentarias y nutricionales que conduzcan a la salud y bienestar, ha sido reconocida como uno de los elementos esenciales para contribuir a la prevención y control de problemas relacionados con la alimentación en el mundo(5). La educación en alimentación y nutrición debe orientarse a potenciar o modificar los hábitos alimentarios, involucrando a todos los miembros de la comunidad educativa incluyendo: niños, padres, docentes y directivos. Educar sobre la necesidad e importancia de una buena alimentación implica, descubrir y erradicar creencias, mitos y conductas erróneas, promoviendo una mayor conciencia sobre las múltiples funciones o roles que juega o debe jugar la alimentación en las distintas esferas de la vida, la salud, los aprendizajes, la producción, distribución y consumo de alimentos(6).

El plan de salud pública de intervenciones colectivas de Norte de Santander ha invertido esfuerzos y recursos en implementar acciones de educación a los grupos vulnerables de las comunidades, en especial, a la población escolarizada, evidenciando que las creencias, valores, costumbres y tradiciones que se han transmitido de generación en generación, contienen un alto potencial de riesgo frente a la conservación y mejoramiento de la salud; situación que implica invertir muchos años en un proceso de educación constante, contextualizado y principalmente autosostenible; por lo cual, surgió la necesidad de crear la Política Pública Departamental "Cátedra de Salud Pública" mediante la Ordenanza 028 del 03 de septiembre de 2003(7).

La cátedra de salud pública como estrategia para la promoción de la salud, ha sido implementada en las instituciones educativas de manera sostenible e integral, con el fin de generar la adopción de estilos de vida y hábitos alimentarios saludables en los niños, niñas, adolescentes y producir cambios en costumbres, creencias, conductas alimentarias, en los docentes y padres de familia a través de un proyecto pedagógico institucional, permitiendo que la educación en salud se comporte como un factor protector para evitar el desarrollo de diferentes patologías, y contribuir al mejoramiento de la calidad de vida, en el cual se genere autodominio en la toma de decisiones en el ámbito individual y colectivo(7).

En Pamplona, a partir del 2013 se inició con el fortalecimiento de las acciones de la cátedra de salud pública, considerando los resultados obtenidos de un proyecto de investigación efectuado en tres instituciones educativas públicas del municipio, cuyo objetivo fue evaluar la efectividad de la cátedra de salud pública en el componente de nutrición(8). Las debilidades identificadas a partir de dicha investigación, han orientado las intervenciones que el Departamento de Nutrición y Dietética de la Facultad de Salud de la Universidad de Pamplona ha propuesto e implementado para el fortalecimiento continuo de la cátedra de salud pública en el ámbito de la alimentación y nutrición. Sin embargo, con base en la revisión de la literatura se ha establecido que no existen estudios bibliográficos que permitan realizar comparaciones con los resultados obtenidos. Por 
lo tanto, se plantea la presente investigación cuyo objetivo es evaluar la aplicabilidad del componente de nutrición y alimentación de la cátedra de salud pública derivada del conocimiento adquirido para la adopción de conductas saludables en una institución educativa pública de Pamplona.

\section{Materiales y métodos}

Se realizó un estudio descriptivo con estudiantes de $7^{\circ}$ a $11^{\circ}$ grado escolar del Instituto Técnico Arquidioscesano San Francisco de Asís, sede Cristo Rey, - Pamplona- en marzo de 2016. El universo correspondió a 151 estudiantes y la muestra fue de 108, la cual fue obtenida a partir de la fórmula de Balestrini, con un intervalo de confianza del $95 \%$ y un margen de error del 5\%.

Los estudiantes seleccionados fueron aquellos quienes habían participado en las intervenciones de fortalecimiento de la cátedra de salud pública realizadas por los estudiantes del programa de Nutrición y Dietética de la Facultad de Salud de la Universidad de Pamplona en semestres académicos anteriores. Se aplicó una encuesta mediante entrevista personal, que contenía 7 preguntas de escala tipo Likert (nunca, rara vez, algunas veces y siempre). El instrumento se ajustó inicialmente a través de la validación por expertos con el fin de valorar su contenido, claridad, comprensión de las preguntas, suficiencia, pertinencia de las variables y factibilidad de aplicación. Además, se elaboró el instructivo que permitió el diligenciamiento correcto del mismo.

\section{Plan de análisis}

Las variables cualitativas fueron tabuladas mediante el programa estadístico SPSS v.23 para establecer las frecuencias de respuesta. Se determinó la variación de respuesta entre hombres y mujeres mediante Análisis de Varianza (ANOVA) y para identificar el grado escolar en el cual hubo mayor o menor aplicabilidad de los conocimientos adquiridos se realizó la prueba de comparaciones múltiples de Tukey.

\section{Consideraciones éticas}

La investigación se consideró sin riesgo de acuerdo a lo estipulado en la Resolución No 8430 del 04 de octubre de 1993 propuesta por el Ministerio de Salud de Colombia, en la cual se establecen las normas científicas, técnicas y administrativas para la investigación en salud(9). La participación de los estudiantes en la investigación fue voluntaria, se obtuvo el consentimiento institucional y asentimiento de los padres de familia.

\section{Resultados}

De 108 estudiantes de séptimo a undécimo grado, 104 fueron incluidos (96.3\%), el 3,7\% rechazó participar en la investigación. Se evidenció una distribución proporcional del 50\%, tanto del género femenino como masculino. La mayor proporción de los encuestados cursaban séptimo grado $28 \%$, el $21 \%$ undécimo grado, $20 \%$ octavo, $17 \%$ noveno, y el $14 \%$ décimo grado.

El 58\% de los estudiantes refirió que algunas veces identifica los alimentos que le son propios a cada persona de acuerdo a la edad, 19\% rara vez, el 14\% siempre los reconoce y el $9 \%$ restante mencionó que nunca lo hace. En cuanto al consumo de una alimentación equilibrada y adecuada según la edad, el 59\% de los estudiantes manifestó que algunas veces la realiza, el $27 \%$ refirió que siempre, 9\% rara vez y el 5\% restante respondió que nunca la práctica. El 63\% de los estudiantes consideró que los conocimientos adquiridos en el desarrollo de la cátedra de salud pública sobre lactancia materna nunca han sido aplicados en el entorno en el cual se desarrollan, mientras que el $36 \%$ de la población mencionó algunas veces y rara vez y sólo el 1\% siempre lo hace.

Del total de los estudiantes encuestados, el 38\% no reconoce los trastornos alimentarios, ni sus síntomas y consecuencias, el $28 \%$ algunas veces lo hace, mientras que el $21 \%$ refirió que siempre los identifica, la proporción restante rara vez los reconoce. Para el aspecto relacionado con procurar tener una alimentación balanceada y saludable a fin de evitar trastornos alimentarios, el $40 \%$ de la población refirió que siempre lo hace, el 35\% algunas veces y el 13\% nunca lo hacían, mientras que el $12 \%$ consideró que rara vez tenían una alimentación saludable. El 54\% de los 
estudiantes afirmaron que algunas veces practican hábitos de higiene con los alimentos que consumen, el $18 \%$ siempre lo hace; el porcentaje restante rara vez y nunca lo practican.

Con respecto a la práctica de las normas de higiene al momento de comprar los alimentos, el $45 \%$ manifestó realizarlas en algunas ocasiones, el 18\% siempre las tiene en cuenta, el 13\% rara vez y $4 \%$ nunca las practica. A partir de lo anterior, se evidenció menor aplicabilidad de los conocimientos en la práctica de una alimentación balanceada y saludable a fin de evitar trastornos alimentarios, así como de los conocimientos adquiridos sobre lactancia materna.

$\mathrm{Al}$ analizar la relación entre el género y los ítems evaluados, se observó una diferencia estadísticamente significativa $(p=0,031)$, en cuyo caso las mujeres aplican con mayor frecuencia los conocimientos adquiridos sobre lactancia materna en el entorno en el cual se desarrollan con respecto a los hombres (Tabla 1).

Tabla 1. Aplicabilidad de los conocimientos adquiridos según género y método paramétrico, Pamplona, 2016

\begin{tabular}{|c|c|c|c|}
\hline \multirow[b]{2}{*}{ Ítems evaluados } & \multicolumn{3}{|c|}{ Género } \\
\hline & $\begin{array}{l}\text { Masculino } \\
\text { Media }\end{array}$ & $\begin{array}{l}\text { Femenino } \\
\text { Media }\end{array}$ & P. Valor \\
\hline Identifica los alimentos que le son propios a cada persona de acuerdo a la edad. & 2,75 & 2,81 & 0,715 \\
\hline Practica una alimentación equilibrada y adecuada con su edad. & 3,08 & 3,1 & 0,895 \\
\hline $\begin{array}{l}\text { Considera que los conocimientos adquiridos en el desarrollo de la cátedra de salud } \\
\text { pública sobre la lactancia materna han sido aplicados en el entorno en el cual se } \\
\text { desarrolla. }\end{array}$ & 1,4 & 1,75 & $0,031^{*}$ \\
\hline Reconoce los trastornos alimentarios así como sus síntomas y consecuencias. & 2,31 & 2,35 & 0,87 \\
\hline $\begin{array}{l}\text { Procura tener una alimentación balanceada y saludable a fin de evitar trastornos } \\
\text { alimentarios }\end{array}$ & 2,85 & 3,15 & 0,13 \\
\hline Practica hábitos de higiene con los alimentos que consume. & 3,33 & 3,19 & 0,296 \\
\hline Practica las normas de higiene al momento de comprar los alimentos & 3,17 & 3,19 & 0,913 \\
\hline
\end{tabular}

*Diferencia estadísticamente significativa

Tabla 2. Aplicabilidad de los conocimientos adquiridos según grado escolar y método paramétrico

\begin{tabular}{|c|c|c|c|c|c|c|}
\hline \multirow{2}{*}{ Ítems evaluados } & \multicolumn{5}{|c|}{ Grado escolar (Media) } & \multirow{2}{*}{ P. Valor } \\
\hline & $7^{\circ}$ & $8^{\circ}$ & $9^{\circ}$ & $10^{\circ}$ & $11^{\circ}$ & \\
\hline $\begin{array}{l}\text { Identifica los alimentos que le son propios a cada persona de acuerdo a } \\
\text { la edad. }\end{array}$ & 3.00 & 2,76 & 2,89 & 2,71 & 2,45 & 0,179 \\
\hline Practica una alimentación equilibrada y adecuada con su edad. & 3,28 & 3,19 & 3,11 & 3,14 & 2,68 & 0,057 \\
\hline $\begin{array}{l}\text { Considera que los conocimientos adquiridos en el desarrollo de la } \\
\text { cátedra de salud pública sobre la lactancia materna han sido aplicados } \\
\text { en el entorno en el cual se desarrolla. }\end{array}$ & 1,86 & 1,43 & 1,5 & 1,57 & 1,41 & 0,263 \\
\hline $\begin{array}{l}\text { Reconoce los trastornos alimentarios así como sus síntomas y } \\
\text { consecuencias. }\end{array}$ & 2,55 & 1,76 & 2,5 & 2,64 & 2,23 & 0,117 \\
\hline $\begin{array}{l}\text { Procura tener una alimentación balanceada y saludable a fin de evitar } \\
\text { trastornos alimentarios }\end{array}$ & 3,38 & 2,9 & 3,06 & 3,14 & 2,45 & $0,029 *$ \\
\hline Practica hábitos de higiene con los alimentos que consume. & 1,93 & 1,48 & 1,78 & 1,64 & 1,77 & 0,296 \\
\hline Practica las normas de higiene al momento de comprar los alimentos & 3,17 & 3,05 & 3,28 & 3,21 & 3,23 & 0,92 \\
\hline
\end{tabular}


Se evidenció diferencias significativas en los estudiantes de séptimo grado $(\mathrm{p}=0,029)$, en quienes se identificó que procuran tener una alimentación balanceada y saludable a fin de evitar trastornos alimentarios en comparación con los demás grados escolares. (Tabla 2).

\section{Discusión}

La evaluación de la aplicabilidad del componente de nutrición y alimentación de la cátedra de salud pública en la institución educativa demuestra que, pese a que se imparten los conocimientos en las temáticas establecidas, los estudiantes no necesariamente efectúan la práctica de dichos saberes en los escenarios en los cuales transcurre su vida cotidiana. La relación entre conocimientos en nutrición y adecuadas prácticas, obtenida de diversos estudios indican que el conocimiento de buenas prácticas nutricionales no necesariamente produce su aplicación; en este sentido, las actitudes son mejores predictores de la conducta alimentaria que los conocimientos (10). Sin embargo, el propósito de la educación alimentaria y nutricional es lograr que los niños, adolescentes y jóvenes desarrollen una capacidad crítica para poder optar por una alimentación saludable en un mundo que cambia rápidamente $\mathrm{y}$ en el cual se observa una continua diversificación de alimentos procesados y una pérdida de estilos de alimentación familiar(11).

Según los resultados obtenidos por San Mauro et al.(12), sólo el 43,5\% de los niños y adolescentes de la muestra estudiada reflejaron hábitos alimentarios compatibles con el patrón dietético mediterráneo, es decir, el 56,5\% restante presentan una baja o media adherencia a la dieta mediterránea y, por tanto, necesitarían mejorar su patrón alimentario para adecuarlo al prototipo mediterráneo. En este contexto, los niños, adolescentes y jóvenes son uno de los grupos prioritarios que deben recibir educación alimentaria y nutricional puesto que son consumidores actuales y futuros, que necesitan adquirir patrones alimentarios saludables y perdurables(5). No obstante, con base en los resultados obtenidos en éste estudio se identifica que sólo el $40 \%$ de los adolescentes encuestados practica una alimentación balanceada y saludable a fin de evitar trastornos alimentarios, identificando que los estudiantes de séptimo grado reflejan mayor aplicabilidad en éste aspecto. En contraste, los resultados obtenidos en el estudio efectuado por Zanirati et al.(13), indican que la mayor permanencia en el ambiente escolar proporciona mejor perfil dietético y favorece la práctica de actividad física entre los escolares, pudiendo así justificar e incentivar la implantación de más programas de escuela integrada.

Considerando los resultados referidos por Carvajal(8), se determina que los estudiantes presentan diferencias por grado escolar en la aplicación de la cátedra en el componente de nutrición. Los estudiantes de sexto grado presentan mejor comportamiento en las prácticas de alimentación equilibrada y adecuada con la edad con respecto a séptimo grado; así como también, los estudiantes de noveno grado reconocen más los trastornos alimentarios, así como sus síntomas y consecuencias con respecto a los de séptimo. Dichas diferencias, indican la necesidad de reforzar los conocimientos impartidos a partir de los módulos de formación, en los que se incluyan ilustraciones y mensajes consistentes con la edad de los niños, adolescentes y jóvenes, así como la evaluación de su utilidad constante en todos los grados escolares, de manera que sean más susceptibles de realizar prácticas con resultados positivos perceptibles.

La escuela tiene un rol significativo en la elaboración de saberes y habilidades de sus estudiantes. Es por ello que, cuanto más integrales y sostenidas sean las intervenciones para desarrollar estilos de vida saludables, más positivos y duraderos serán sus efectos(11). Según lo reportado por Quirós y Torres(14), con respecto a la opinión que tienen los estudiantes acerca de la importancia de la enseñanza de una alimentación correcta en el colegio, el 95\% de la población participante, manifiesta que sí es importante que en el colegio se les enseñe a alimentarse correctamente, además argumentan que esto les permite prevenir enfermedades, tener una buena salud y adquirir conocimientos sobre nutrición. Según lo referido en el estudio de 
Yepes et al.(15), la mayoría de los profesionales manifiestan que, especialmente en ciertas situaciones, operarían acuerdos tácitos con la familia, pues es el eje principal en la toma de decisiones.

Es importante introducir la educación nutricional como parte de la enseñanza desde la infancia para la prevención de los trastornos alimentarios, dando lugar a la adquisición de conocimientos actuales y fiables acerca de alimentación, nutrición y salud. Con base en los resultados reportados por Fajardo et al.(16), el riesgo para presentar trastornos de la conducta alimentaria se observó en el $30,1 \%$ de los estudiantes que conformaron la muestra de su estudio, siendo mayor la prevalencia de riesgo en las mujeres, por lo cual se determina que cobra interés el focalizar intervenciones preventivas o de acción en instituciones educativas y diseñar estrategias de salud pública que contribuyan al manejo de esta patología emergente en población infantil y juvenil.

Trabajando de forma conjunta los temas psicológicos y nutricionales se conseguirá el mejoramiento de la capacidad de interpretación de la información ofrecida por medios de comunicación, así como los conocimientos para la elaboración de una alimentación completa, variada y equilibrada seleccionando alimentos de buena calidad nutricional(17).

Así mismo, el 63\% de los estudiantes encuestados considera que los conocimientos adquiridos en el desarrollo de la cátedra de salud pública sobre lactancia materna nunca han sido aplicados en el entorno en el cual se desarrollan, destacándose las mujeres como aquellas que aplican mayoritariamente dicho conocimiento. Éste resultado coincide con lo reportado por Carvajal(8), en el cual se demuestra que la aplicación de los conocimientos sobre lactancia materna constituye uno de los aspectos con valores menos favorables, lo cual se encuentra interrelacionado con los objetivos que no fueron objeto de cumplimiento según lo consideran los docentes, específicamente en los contenidos temáticos del módulo de adolescencia dadas las características de los estudiantes encuestados $\left(6^{\circ}\right.$ a 9ㅇ).

Con base en los resultados referidos por Forero et al.(18), se observa que las adolescentes tienen conocimientos claros del discurso biomédico sobre los beneficios de la lactancia materna, sin embargo, este conocimiento se manifiesta de diferentes formas en las experiencias particulares de cada una de ellas. Por tanto, el estudio y la comprensión del adolescente en todas sus dimensiones (física, biológica, psicológica, social, cultural, genérica, sexualidad, comunicación intra y extrafamiliar, sistema educativo formal, sistema de salud, entre otras), le permiten asumir una posición frente a la práctica de la lactancia natural, deben ser analizadas globalmente si se desean encontrar respuestas integrales al por qué los adolescentes asumen diferentes actitudes y tienen diferentes conocimientos con respecto a la lactancia natural. La disponibilidad de información oportuna y confiable es un aspecto fundamental para el desarrollo de estrategias efectivas y acciones de fomento de la lactancia natural. El análisis de esta información facilita la identificación de características específicas y singulares que apoyen la implementación de políticas y planes de acción acordes con los conocimientos, las actitudes, necesidades e intereses de la población adolescente(10).

De ésta manera, aun cuando el componente de nutrición y alimentación de la cátedra de salud pública está diseñado con objetivos y contenidos definidos, los resultados indican la importancia de identificar las características de la población no sólo a partir de los conocimientos sino también desde el ámbito económico y social, con el objeto de incidir de forma coherente en la aplicación de los saberes que se imparten; además, las intervenciones de educación en nutrición deben ser parte de programas transversales orientados a mejorar la salud, nutrición, y medio ambiente, generando a partir de futuras investigaciones procesos de evaluación continua que reorienten las estrategias en un contexto de desarrollo socioeconómico que contribuya al bienestar y la calidad de vida de la población. 


\section{Conclusiones}

Se identificaron falencias en la aplicabilidad de los conocimientos del componente de nutrición y alimentación adquiridos a partir de la cátedra de salud pública, lo cual determina la necesidad de implementar estrategias de educación eficaces que destaquen la importancia de la nutrición y alimentación del adolescente, con el fin de favorecer la adopción de prácticas saludables así como evitar posibles factores de riesgo de determinadas patologías propias de los adultos y de gran importancia desde el punto de vista sanitario.

Es necesario insistir en la trascendencia de la institución educativa como un entorno ideal para el desarrollo de acciones de promoción de la salud y prevención de enfermedades, en el cual, se aborden procesos de aprendizaje integrales de bienestar y calidad de vida, dada su influencia en las etapas formativas en las cuales, la familia y la comunidad también deben ser parte fundamental de las orientaciones e intervenciones en éste aspecto.

\section{Agradecimientos}

A los estudiantes de décimo semestre del programa de Nutrición y Dietética que cursaron la Práctica Campos de Acción Profesional por su participación en la recolección de la información. A los estudiantes y docentes de la institución educativa que participaron en este estudio.

\section{Conflicto de intereses}

Las autoras declaran que no existen conflictos de intereses.

\section{Referencias}

1. Castillo-Reinosa M. La nutrición en el marco de la educación para la salud, un instrumento para incidir en el aumento de resiliencia y en el proyecto de vida del educando. Bogotá: Universidad Nacional de Colombia; 2012.

2. Riquelme Pérez M. Educación para la salud escolar [Internet]. Madrid: Exlibris Ediciones; 2006. Available from:

https://www.aepap.org/sites/default/files/educacion_ salud_escuela.pdf

3. Pedrero E, Morón JA. Aproximación al concepto de Educación para la Salud : Una Perspectiva Histórica. In: I
Congreso Virtual Internacional sobre Innovación Pedagógica y Praxis Educativa INNOVAGOGÍA [Internet]. 2012. p. 1594-601. Available from: https://dialnet.unirioja.es/servlet/articulo? codigo $=466$ 5661

4. Eslava-Castañeda JC. Repensando la promoción de la salud en el sistema general de seguridad social en salud. Rev Salud Pública [Internet]. 2006;8(Supl 2):106-15. Available from: http://www.scielosp.org/pdf/rsap/v8s2/v8s2a09.pdf

5. Olivares S, Snel J, Glasauer MMP. Educación en nutrición en las escuelas primarias. Educ en Nutr en las Esc primarias. 1998;22(Cuadro 1):57-62.

6. De La Cruz E. La Educación Alimentaria y Nutricional en el contexto de la Educación Inicial. Paradigma [Internet]. 2015;XXXVI:161-83. Available from: http://revistas.upel.edu.ve/index.php/paradigma/artic le/view/2663/1275

7. Torres I, Katherine C. Guía metodológica de la Cátedra de Salud Pública. Ocaña: Departamento Norte de Santander; 2013.

8. Carvajal-Suárez LS. Evaluación de la efectividad de la cátedra de salud pública, componente de nutrición, Municipio de Pamplona, Departamento Norte de Santander - Colombia, 2012. Bogotá: Universidad de Los Andes; 2012.

9. Ministerio de Salud. Resolución 8430 de 1993. Bogotá: MinSalud; 1993.

10. Dávila CG, Gutiérrez LG. Conocimientos y actitudes de la población adolescente hacia la lactancia materna para direccionar las acciones estrategias de promoción de la lactancia natural. Costa Rica: Universidad Estatal a Distancia; 2004. Available from: http://repositorio.uned.ac.cr/reuned/handle/120809/ 1085

11. Ministerio de Educación. Educación Alimentaria y Nutricional Saludable. Argentina: Gobierno de la Provincia de Córdoba; 2013. Available from: http://www.nutrinfo.com/biblioteca/libros_digitales/e du_Educ_Ali_Nutr_Escuela.pdf

12. San Mauro I, Megias A, García de Angulo B, Bodega $P$, Rodríguez $\mathrm{P}$, Grande $\mathrm{G}$, et al. Influencia de hábitos saludables en el estado ponderal de niños y adolescentes en edad escolar. Nutr Hosp [Internet]. 2015;31(5):1996$2005 . \quad$ Available from: http://www.ncbi.nlm.nih.gov/pubmed/25929367

13. Zanirati VF, Lopes ACS, Santos LC Dos. Contribuição do turno escolar estendido para o perfil alimentar e de atividade física entre escolares. Rev Panam Salud Publica [Internet]. 2014;35(1):38-45. Available from: http://www.scielosp.org/scielo.php?script=sci_arttext \&pid=S1020-49892014000100006\&lng=en\&nrm=iso

14. Quirós-Rojas JF, Torres-Salas MI. La enseñanza de la nutrición a nivel de secundaria utilizando el tema transversal "educación para la salud" desde un enfoque útil para la vida. Rev Electrónica Educ [Internet]. 2015;19(2):1-20. Available from: http://www.revistas.una.ac.cr/index.php/EDUCARE/ar ticle/view/6572

15. Yepes T, Alzate-Cánovas LP. Estado nutricional infantil y estilos educativos familiares: apreciación de expertos. 
Perspect en Nutr Humana [Internet]. 2013;15:185-99. Available

from: http://www.scielo.org.co/pdf/penh/v15n2/v15n2a6.p df

16. Fajardo E, Méndez C, Jauregui A. Prevalencia del riesgo de trastornos de la conducta alimentaria en una población de estudiantes de secundaria, Bogotá Colombia. Rev Med [Internet]. 2017;25(1):46-57. Available from: https://revistas.unimilitar.edu.co/index.php/rmed/arti cle/view/2917

17. Bolaños-Ríos P. La educación nutricional como factor de protección en los trastornos de la conducta alimentaria. Trastor la Conduct Aliment ISSN-e 1699-7611, No 10, 2009, págs 1069-1086. 2009;10(10):1069-86.

18. Forero $\mathrm{Y}$, Isaacs $\mathrm{M}$, Rodríguez $\mathrm{S}$, Hernández J. La lactancia materna desde la perspectiva de madres adolescentes de la ciudad de Bogotá. Biomédica [Internet]. 2013;33(4):554-63. Available from: http://www.revistabiomedica.org/index.php/biomedic a/article/view/1470 